\title{
EL ÁFRICA ORIENTAL ITALIANA Y EL DESARROLLO DE UNA ANTROPOLOGÍA NACIONAL, 1890 - 1941
}

\author{
THE EASTERN ITALIAN AFRICA AND THE DEVELOPMENT OF A NATIONAL \\ ANTHROPOLOGY, 1890 - 1941
}

Diego Giovanni Castellanos ${ }^{1}$ Universidad Nacional de Colombia DOI: 10.17533/udea.tempus.n8a04

\section{Resumen}

El texto busca identificar y analizar la relación que pudo haber tenido el surgimiento y desarrollo de la disciplina antropológica en Italia con la estrategia de expansión colonial dirigida por el Estado italiano, en el periodo que va de 1890 a 1941. Como se muestra, en un primer momento los antropólogos manifestaron más bien poco interés por las realidades coloniales, al punto que los estudios necesitados por el Estado se basaron en informaciones parciales y estereotipadas, producto del trabajo de funcionarios y no de académicos. Sin embargo, posteriormente la política del régimen fascista colocó los estudios antropológicos en el centro del debate público y académico, debido a la necesidad de justificar ideológicamente las acciones del Estado y la expansión imperial. Se considera que dicho interés usualmente fue utilitarista, siendo que se suponía que un mejor conocimiento de las poblaciones ocupadas facilitaría su dominación, pero también surgieron propuestas e interpretaciones que incluso en ocasiones fueron en contra de los postulados del estado.

Palabras clave: Colonialismo italiano, racismo, historia de la antropología.

\begin{abstract}
The text identifies and analyzes the relation that could exist among the emergence and development of the anthropological discipline in Italy and the strategy of colonial expansion directed by the Italian State, in the period from 1890 to 1941. At first, the anthropologists expressed slight interest in the social realities of the colonies, to the point

\footnotetext{
${ }^{1}$ Candidato a doctor en Historia, Universidad Nacional de Colombia. Investigador del Grupo de Estudios Sociales de la Religión. gcastellanosd@unal.edu.co
} 
TEMPUS. Revista en Historia General Medellín (Colombia), 2018, septiembre-octubre, número 8

that the studies required by the State were based on incomplete and stereotyped information, the product of the activity of officials and not by specialists. Later the policy of the fascist regime placed anthropological studies at the center of the public and academic debate, due to the necessity to justify ideologically the efforts of the imperial expansion. We consider that this interest was usually utilitarian since it was assumed that a better knowledge of the occupied populations could strengthen their control. However, also arose approaches and arguments that even went against the postulates of the Fascist regime.

Keywords: Italian colonialism, racism, history of anthropology.

"Occupare la terra per vivere è di diritto naturale delle genti. Occuparne tanta quanta può servire, non solo ai bisogni attuali, ma a quelli ragionevolmente prevedibili della stirpe in via di sviluppo, è ancora un diritto".

Rodolfo Benini, Le ragioni dell'Italia ${ }^{2}$

Este artículo tiene como propósito señalar la relación entre el establecimiento de Italia como potencia colonial y el surgimiento de la antropología en dicho país. Como tal se busca identificar y analizar la relación que tuviera el discurso del estado italiano dirigido a legitimar su dominación sobre territorios africanos, especialmente en el cuerno de África, y el desarrollo teórico de la disciplina, tal como fue realizada por los investigadores y los institutos más prestigiosos. En un contexto de predominio de un paradigma evolucionista, el texto se enfoca en el periodo que va de 1890, con el inicio de la colonización de Eritrea, y culmina en 1941, con el fin de la aventura colonial italiana.

\footnotetext{
${ }^{2}$ Rodolfo Benini, "Le ragioni dell'Italia”, Giornale degli Economisti e Rivista di Statistica 76, n. 1 (1936).
} 
El texto se refiere a un momento de la historia de la república italiana en el cual, tras la unificación, el Estado y de algunos sectores ilustrados de la población consideraron importante la expansión colonial con miras a asegurarse un lugar entre las naciones civilizadas. Igualmente, el artículo aborda un momento del desarrollo de una disciplina particularmente moderna y colonial, la antropología.

La hipótesis manejada por autores como Lanternari ${ }^{3}$ es que no hubo relación entre antropología y colonialismo en el caso italiano, puesto que no habría realmente desarrollo de la antropología social en Italia hasta el fin de la Segunda Guerra Mundial. Sin embargo, diversos autores también han llamado la atención acerca de la necesidad de vincular las raíces del ejercicio antropológico italiano a la experiencia colonial. Autores como Puccini y Squillacciotti, así como Leone, han establecido la existencia de un fuerte nexo entre el desarrollo de la antropología italiana y el régimen fascista. Aún más, Bárbara Sorgoni ha indicado la necesidad de observar la manera en la que el colonialismo se vio afectado por el desarrollo de la disciplina. ${ }^{4}$ En dicho sentido, el ensayo sigue la línea de los autores como los anteriormente mencionados, quienes afirman que la antropología si tuvo que ver con la descripción y clasificación de las poblaciones africanas que vivían bajo mandato italiano. ${ }^{5}$

Mia Fuller sostiene que el tema del colonialismo italiano no ha recibido tanta atención por parte de los académicos como debiera. En parte, atribuye este hecho a las circunstancias que marcaron el fin de este fenómeno, que llegó a su fin de manera abrupta con la terminación de la guerra y la caída del régimen. Esto condujo a que muchos documentos y archivos permanecieran escondidos o ignorados durante décadas, antes de que empezaran lentamente a ver la luz. ${ }^{6}$ Otra razón, acaso más importante, es el hecho resaltado por Claudio Pavone, quien afirma que tras la guerra

\footnotetext{
${ }^{3}$ Vittorio Lanternari, Antropologia e Imperialismo (Turin: Einaudi, 1974), 322.

${ }^{4}$ Patrizia Palumbo, A Place in the Sun. Africa in Italian Colonial Culture from PostUnification to the Present (Berkeley \& Los Angeles: University of California Press, 2003).

${ }^{5}$ Barbara Sorgoni, "An Italian officer in colonial Eritrea: administration, ethnography and gender", en Ordering Africa. Anthropology, European imperialism and the politics of knowledge, de H. Tilley y R. J. Gordon (Manchester: Manchester University Press, 2007), 330-374.

${ }^{6}$ Ruth Ben-Ghiat y Mia Fuller, Italian Colonialism (Nueva York: Palgrave Macmillan, 2005), 2.
} 
TEMPUS. Revista en Historia General Medellín (Colombia), 2018, septiembre-octubre, número 8

se desarrolló un sentimiento de vergüenza frente a la política racista en las colonias, lo que se sumó a la ya de por sí relativa falta de interés que sobre el pasado y presente de dichos territorios poseía una gran parte de la población. ${ }^{7}$

A dicho sentimiento, Mia Fuller añade la falta de interés académico por profundizar en los registros del pasado colonial, en un contexto de mutua recriminación entre partidarios del fascismo y del comunismo, en las décadas inmediatamente siguientes a la caída del régimen fascista. Incluso a pesar de que estudios sobre la experiencia colonial comenzaron a surgir desde finales de la década de los 50, aun así existían limitaciones de acceso a documentación que dificultaban la tarea. Así, verdaderas síntesis comprehensivas del fenómeno comenzaron a aparecer solo en los últimos 15 años. ${ }^{8}$

Un hecho resaltable de este reciente interés ha sido el acercamiento entre historiadores y antropólogos, que sirve como indicador del cambio de actitud. Pese a esto, Claudio Pavone llama la atención al hecho de que, incluso recientemente, dichos estudios han provocado "bitter reactions on the part of the traditionalist historians who had appointed themselves custodians of what they called Italy's 'good reputation' in Africa". 9

En dicho orden de ideas, en este texto se parte de la hipótesis inicial de que, pese a la predominancia de un enfoque biológico entre quienes ejercían la disciplina en Italia, los antropólogos si llegaron a interesarse por las poblaciones africanas que habitaban los territorios dominados. Se considera que dicho interés usualmente fue utilitarista, siendo que se suponía que un mejor conocimiento de las poblaciones ocupadas facilitaría su dominación, pero también surgieron propuestas e interpretaciones que incluso en ocasiones fueron en contra de los postulados del estado. Así, pese al más bien limitado desarrollo alcanzado por la antropología italiana en comparación con la realizada por otras potencias coloniales, se considera que el trabajo realizado sobre las poblaciones sometidas marcó el desarrollo de la disciplina en dicho país.

\footnotetext{
${ }^{7}$ Caludio Pavone, "The Two Levels of Public Use of the Past", Mediterranean Historical Review, n. 16 (2001): 74-86.

${ }^{8}$ Ben-Ghiat y Fuller, Italian..., 3.

${ }^{9}$ Pavone, "The Two Levels of Public Use of the Past", 74-86.
} 
Tal como afirman Burbank y Cooper, los imperios coloniales buscaron conscientemente enfatizar la diferencia entre quienes estaban a cargo de construir el imperio sobre el territorio colonizado y las poblaciones nativas, recurriendo a discursos de racialización. Oponiéndose a las tendencias sociales en contra, se buscó establecer límites, espacios diferenciados y clasificaciones del tipo "nosotros/ellos". ${ }^{10}$ Valga traer a colación a Edward Said, cuando llamaba la atención acerca de la necesidad de identificar la manera estereotipada y prejuiciosa en la que el saber europeo se acercó a las poblaciones no occidentales, presentando al otro como estático en el tiempo e inferior, con lo que se vino a justificar su dominación. ${ }^{11}$

\section{La Experiencia Colonial Italiana}

El colonialismo, el fenómeno por el cual una nación busca ejercer dominio sobre otras gentes o territorios, usualmente con fines de explotar sus recursos, fue un fenómeno que en su faceta moderna se presentó como un hecho principalmente europeo sobre otros continentes. En el caso italiano, dicha política estuvo relacionada con la posesión de territorios principalmente en África del norte y oriental.

La experiencia colonial italiana se ha dividido en dos grandes etapas: el periodo del estado liberal, que se inicia en 1869, poco después de la unificación, y el periodo fascista, de 1928 a 1941. Aunque se presentaron diferencias perceptibles en la política interna italiana respecto a las colonias entre ambos periodos, para la población colonizada al parecer dichos cambios no fueron evidentes, salvo por el inicio de nuevas hostilidades cuando se procuró ampliar el territorio.

Durante el primer periodo fue posible advertir un interés por aprovechar la temprana presencia de algunas entidades privadas en África Oriental como parte de una política expansiva de la nueva Italia unificada. Ya Giussepe Mazzini (1805-1872), el renombrado político y activista italiano, consideró la presencia italiana en África como

\footnotetext{
${ }^{10}$ Jane Burbank y Frederick Cooper, Empires in world history. Power and the politics of difference (Princeton: Princeton University Press, 2010), 12.

${ }^{11}$ Priyansh Ranjan, “Edward Said's 'Orientalism': A Post-Colonial Culture Study”, IOSR Journal of Humanities and Social Science 20, n. 9 (2015): 85-88.
} 
TEMPUS. Revista en Historia General Medellín (Colombia), 2018, septiembre-octubre, número 8

ISSN: 2422-2976

importante en relación con la "misión" de Italia en el mundo (Duggan 1996, 239); y así mismo Francesco Crispi (1818-1901), quien fuera Presidente del Consejo de Ministros entre 1887 y 1891, y nuevamente de 1893 a 1896, "en un intento por salvar la nación y de paso su propia reputación" vio oportuna la creación de colonias en África. ${ }^{12}$ Otras causas que se utilizaron para justificar el colonialismo fue la presión demográfica en el país, la intención de crear colonias penales, y encarar la que se conocía como la "questione meridionale". En efecto, tal como afirma Christopher Duggan, para finales del siglo XIX y comienzos del XX, el área rural italiana estaba superpoblada, las oportunidades económicas eran limitadas $\mathrm{y}$, especialmente en el sur, la mayor parte de la población vivía en áreas rurales rozando la subsistencia. ${ }^{13} \mathrm{Sin}$ embargo, el mayor atractivo para quienes emigraban eran los países americanos, por lo que el impacto de las colonias en la resolución de este problema fue casi imperceptible.

El antecedente inmediato de la presencia colonial tuvo lugar cuando, en 1869, el país adquirió algunas pequeñas posesiones de tierra sobre la costa del Mar Rojo, en un momento en el cual aún no se había logrado integrar efectivamente Roma a la Italia unificada. De hecho, en su primera etapa (1869-1885) el Estado italiano no se involucró en primera persona, sino que recurrió a intermediarios, siendo de hecho Giuseppe Sapeto el primer administrador de los nuevos territorios cuando recibió la bahía tras la derrota del sultán de Raheita. El acto se llevó a cabo como una compra realizada por la compañía mercante Rubattino, adquiriéndose posteriormente nuevas posesiones, hasta que el Estado italiano concedió al territorio el estatus de colonia en 1882. ${ }^{14}$ Sin embargo, el colonialismo italiano comenzó como tal en la década de 1890 , como reacción a que Francia hubiera arrebatado Túnez al Imperio Otomano en 1882, lo que fue interpretado como la "pérdida" de una potencial colonia italiana, dada la presencia relativamente importante de italianos en dicho territorio. 15

\footnotetext{
${ }^{12}$ Charlotte Volpe, Imagined Proximities: The Use of History and Geography as Rhetorical Justifications for Italian Colonial Conquest in Tripolitania (Nueva York: Barnard College, 2014), 16.

${ }^{13}$ Christopher Duggan, Historia de Italia (Cambridge: Cambridge University Press, 1996), 248.

${ }^{14}$ Giuseppe Stefanini, "Assab", Enciclopedia italiana di scienze, lettere ed arti, de Istituto Giovanni Treccani (Roma: Istituto della Enciclopedia italiana, 1929).

${ }^{15}$ Ben-Ghiat y Fuller, Italian..., 2.
} 
En el caso de África Oriental, en la década de 1880 Italia había ocupado el puerto de Massawa, en la costa del Mar Rojo, pero no logró ir más adentro en el territorio ni hacerse con el comercio local. Sin embargo, se adquirieron pequeños territorios en lo que más adelante sería el Estado de Somalia. Los enfrentamientos con la población local continuaron y en 1887 un enfrentamiento en Dogali tuvo como resultado quinientos soldados italianos muertos. Otro percance se presentó cuando un tratado por el cual el Emperador de Abisinia reconocía Etiopia como protectorado italiano quedó anulado por un error de traducción. ${ }^{16}$ Estos sucesos eran seguidos por la población italiana y tuvieron efectos internos. A tal punto que la Batalla de Adowa, que tuvo lugar el 1 de marzo de 1896 y en la que murieron 5.000 soldados italianos, fue considerada la peor derrota de una potencia colonial en África y ocasionó el desplome político de Francesco Crispí.

En el segundo periodo, Benito Mussolini había prometido la creación del Imperio Italiano, que controlaría el Mediterráneo. Sin embargo, ante la fuerza de Francia y el Reino Unido en la región, se resolvió por la invasión de Etiopia, con el fin de aumentar el prestigio italiano y hacer contrapeso a problemas internos. Al mismo tiempo, simbólicamente se quería tomar revancha de la Batalla de Adowa, con el agravante de que en dicho momento Etiopia era miembro de la Sociedad de Naciones. Este organismo condenó a Italia como país agresor en 1935, pese a lo cual Italia anexiono oficialmente el territorio etíope el 7 de mayo de 1936, en una campaña caracterizada por el uso de armas químicas y la masacre de clérigos. Fue así como se dio inicio al "Imperio Italiano", que en África Oriental incluyó Eritrea, parte de Somalia y Etiopía. Sin embargo, Italia perdió todos los territorios coloniales en 1941, realidad reconocida oficialmente por el Estado en 1947.

\section{Antropología y colonialismo en Italia}

Una vez establecidas las colonias en África Oriental, surgió la necesidad por parte del Estado por proveerse de información más o menos fidedigna acerca del territorio y sus habitantes. Esto no era fácil, ya que para finales del siglo XIX no había demasia-

\footnotetext{
${ }^{16}$ Duggan, Historia..., 239.
} 
TEMPUS. Revista en Historia General Medellín (Colombia), 2018, septiembre-octubre, número 8

ISSN: 2422-2976

dos académicos capacitados que viajaran a las colonias y desarrollaran investigaciones en campo. No solo la antropología aún no había centrado sus metodologías en el trabajo etnográfico, sino que los nuevos territorios ofrecían poco atractivo para quienes ejercían la naciente disciplina.

Frente a esto, los datos que se recopilaron durante las primeras décadas provenía de la información obtenida por parte de oficiales coloniales, viajeros, e informes de funcionarios, por lo que puede decirse que la descripción y clasificación inicial de los pueblos nativos fue realizada de manera burda y superficial, basada en impresiones iniciales. ${ }^{17}$ Sin embargo, dichas descripciones y categorías iniciales fueron exitosas dentro de la sociedad italiana, y se mantuvieron como referentes durante la mayor parte del periodo colonial.

La falta de interés a la cual se hacía referencia se debía a la manera en la que por entonces se percibía a la joven disciplina en Italia, que durante esos años se enfocaba principalmente en la antropología biológica, y especialmente al estudio de huesos y cuerpos. Estos eran recolectados en lugares distantes, trasladados a la metrópoli, y posteriormente armados, catalogados y usados para establecer patrones raciales y contribuir a su ubicación en un patrón evolutivo de la especie. Los antropólogos trabajaban sobre un mismo supuesto de que existía una relación entre las características físicas, mentales y psicológicas, con lo que situaban a los pueblos en diferentes niveles de civilización.

Por supuesto, seria anacrónico culpar de aproximaciones malintencionadas a quienes formularon estas clasificaciones. Tal como sostiene Juan Goberna "la perspectiva evolucionista dominaba claramente el pensamiento antropológico de la época" ${ }^{18}$ Valga recordar que Lewis Morgan publicó en 1877 la Sociedad Primitiva, en la que proponía el estudio de la historia humana según el paso por etapas sucesivas que iban del salvajismo a la barbarie, y de ésta a la civilización. Morgan afirmaba: "la inferioridad del hombre salvaje en la escala mental y moral, sin desarrollar, sin experiencia y man-

\footnotetext{
${ }^{17}$ Sorgoni, “An Italian officer in colonial Eritrea: administration, ethnography and gender",62.

${ }^{18}$ Juan Goberna Flaque, "What's culture: Cien años de controversias en la antropología anglosajona (1871-1971)", Gallaecia, n. 22 (2003): 531-554.
} 
tenido por sus bajos apetitos animales y pasiones... es sustancialmente demostrado por los restos del arte antiguo... se ilustra demás por la condición presente de tribus salvajes en un estado bajo de desarrollo". ${ }^{19}$

No solo en Italia, sino incluso en instituciones académicas inglesas, la palabra antropología era utilizada con un sentido biológico más que cultural, siendo de hecho Edward Burnett Tylor (1832-1917) quien la utilizó en primer lugar en un sentido de estudio de la cultura. No hay que olvidar que fue solo en 1871 que Primitive Culture, su obra maestra, fue publicada, cuando el proyecto colonial italiano ya estaba en marcha. En Primitive Culture Tylor sostenía la similitud básica de todas las mentes humanas, proponiendo la unidad psíquica de la humanidad, al tiempo que establecía un esquema evolucionista de la religión según surgía del animismo, pasaba al politeísmo y llegaba al monoteísmo. Según Tylor "esta hipotética situación primitiva corresponde, notablemente, a la de las modernas tribus salvajes". ${ }^{20}$

Tal como afirma Marvin Harris, el desarrollo de dicho determinismo racial decimonónico tenía su raíz en la obsesión con el progreso y lo caracterizaba la preocupación por demostrar la transmisión hereditaria de diferencias raciales en la aptitud para crear, adquirir o alcanzar la civilización. ${ }^{21} \mathrm{Y}$ si bien los antropólogos solían evitar relaciones fáciles o simplistas entre raza y cultura, su idea de evolución cultural era tomada superficialmente por el pensamiento estatal y la opinión pública de la época.

Cabe además tener en cuenta que fue ese un periodo en el que los antropólogos italianos en su mayoría no viajaban, sino que trabajaban sobre restos humanos y productos manuales recolectados por oficiales y viajeros de la institución colonial, en cercana relación a los nuevos museos fundados por todo el país. Se consideraba que personas con poca preparación estaban capacitadas para recoger información en campo y enviarla a los verdaderos especialistas. Este tipo de división del trabajo se mantuvo hasta el final del periodo fascista: los antropólogos escribían guías para indicar a los

\footnotetext{
${ }^{19}$ Lewis Morgan, La Sociedad Primitiva (Buenos Aires: Lautaro, 1946), 109.

${ }^{20}$ Edward Tylor, Cultura Primitiva: Los orígenes de la cultura (Madrid: Ayuso, 1977), 41.

${ }^{21}$ Marvin Harris, El desarrollo de la teoría antropológica: Una historia de las teorías de la cultura (Madrid: Siglo Veintiuno de España Editores, 2005), 71.
} 
TEMPUS. Revista en Historia General Medellín (Colombia), 2018, septiembre-octubre, número 8

ISSN: 2422-2976

viajeros y oficiales como recoger información que se consideraba relevante. Sin embargo, hubo excepciones a esta regla, aunque en realidad fueron más bien pocos los académicos que se establecieron en las colonias, o que por lo menos viajaron a recoger ellos mismos la información que utilizaron.

\section{Antropología y Estado Colonial}

Una vez decidida la invasión de Etiopia, el Estado italiano buscó la movilización de la sociedad en apoyo a la empresa colonial, dentro de la cual se esperaba la participación de científicos. La respuesta de éstos fue más bien tímida, tal como afirma Roberto Maiocchi, y los únicos que manifestaron un interés especial fueron aquellos que poseían un interés colonial previo y vieron la posibilidad de ganar prestigio y reconocimiento. Algunos de dichos académicos fueron después firmantes del Manifesto della $R a z z a{ }^{22}$ y defendieron la expansión colonial como un "problema científico". ${ }^{23}$

En general, los académicos italianos no poseían mayor información acerca de los recursos o las poblaciones que se disponían a colonizar, e incluso de algunas ya colonizadas, razón por la cual tendían a ofrecer informaciones superficiales y repetitivas. En un artículo de este corte publicado en septiembre de 1935, Conti Rossini sostenía que Abisinia era incapaz de lograr por si misma algún progreso, con lo que justificaba su conquista y dominación. ${ }^{24}$

Cuando Eritrea se convirtió oficialmente en la primera colonia italiana, esta poseía unos 250.000 habitantes $^{25}$ distribuidos en unos $110.000 \mathrm{~km} 2$. Por ser la primera expe${ }^{22}$ Fue una disertación académica publicada en 1938, en colaboración de intelectuales y profeso-
res universitarios bajo el régimen fascista, en la que se establecía la primacía racial, biológica y
cultural, así como la eugenesia y la estratificación social, como bases para la civilización. Una
vez convertido en ley en 1939, el manifiesto permitió al estado legitimar el dominio sobre etío-
pes, albanos y árabes como biológicamente inferiores. Ver: "Razzismo Italiano", La Difesa
della Razza 1 , n. 1 (1938).
${ }^{23}$ Maiocchi Roberto, "Gli scienziati italiani e la guerra d'Etiopia", Culture Economie e
Territori, n. 32 (2012): 32 .
${ }^{24}$ Carlo Conti-Rossini, "L'Etiopia è incapace di progresso civile", Nuova antologia (1935):
$342-356$.
${ }^{25}$ De los cuales, según datos de la Enciclopedia Italiana, eran musulmanes el 55\%; cristianos
monofisitas $37,41 \%$; católicos $2,64 \%$; protestantes 0,11\%; y paganos $4,49 \%$. El número de
población italiana era, pese a los esfuerzos del Gobierno colonial, más bien escaso: 3874 para
1921 . Ver: Attilio Mori, Enrico Cerulli, Giuseppe Stefanini, Fabrizio Cortesi, Mario Salfi, y
Ana Maria Ratti, "Eritrea, Colonia", Enciclopedia italiana di scienze, lettere ed arti (Roma: Ist.
della Enciclopedia Italiana, 1932). 
riencia de dominación republicana, Eritrea se convirtió en el laboratorio para el desarrollo una política colonial y, mediante el trabajo de Carlo Conti Rossini, se impulsó la clasificación de la población según tres grupos con diferente grado de desarrollo:

- Cristianos coptos de las tierras altas, que representaban el mayor grupo en el territorio, pertenecientes principalmente a la etnia Tigrinyan, dedicada esencialmente a la agricultura.

- Grupos calificados como "aristocráticos", que en general eran grupos pastoriles o semi pastoriles de religión musulmana, con una fuerte estructura social y ubicados al norte del territorio. Estos estaban representados por las etnias Habab, Marya, Mensa, Bilen y Afar.

- Musulmanes "democráticos", ubicados al sur-occidente. A pesar de estar dedicados a la agricultura, por mostrar un marcado igualitarismo tribal se les consideraba más primitivos. Estaban representados por las etnias Kunama, Nara y Saho. ${ }^{26}$

Además de ser provisional y reduccionista, esta clasificación se impuso en buena medida por el prestigio del mismo Rossini, considerado en su época uno de los más destacados "etiopistas". Nacido en Salerno en 1872 y muerto en Roma en 1949, Rossini permaneció cinco años en Eritrea, entre 1899 y 1903, desempeñándose como funcionario en el cargo de director de asuntos civiles y políticos. A pesar de ser reconocido especialmente como geógrafo y cartógrafo, Rossini ejerció una influencia notoria en el conocimiento de los pueblos colonizados mediante su obra "Principi di diritto consuetudinario dell'Eritrea", de 1916. La suya es una visión evolucionista de la sociedad, según la cual las sociedades humanas presentaban diferentes grados de desarrollo, lo cual, de alguna manera legitimaba la dominación de unos pueblos sobre otros. $^{27}$

\footnotetext{
${ }^{26}$ Valerio Panza, La colonizzazione giuridica dell'Eritrea: diritto coloniale tra scienza giuridica, antropologia, etnografia giuridica 1880-1912 (Nápoles: Università Degli Studi Di Napoli "Federico II", 2014).

${ }^{27}$ Queste due genti affini dell'Eritrea occidentale hanno dunque una struttura sociale che ricorda, se non il matriarcato, come alcuni hanno detto, almeno un ordinamento gentilizio entro il quale il nucleo familiare non si è più ben differenziato. Naturalmente un tale stato può essere
} 
TEMPUS. Revista en Historia General Medellín (Colombia), 2018, septiembre-octubre, número 8

ISSN: 2422-2976

Tal como se afirmó anteriormente, estos conceptos no eran particulares de Italia, sino que correspondían a un momento en el desarrollo de las Ciencias Sociales en Europa, de allí también su grado de aceptación. Esto se evidencia en una reseña francesa a la obra de Rossini, en la que se apoya la necesidad de clasificar a los grupos humanos en categorías jerárquicas según su grado de evolución cultural:

"grâce à sa vaste érudition, il a esquissé, autant qu'il était possible de le faire dans l'état actuel de nos connaissances, l'histoire de ces mêmes tribus et les diverses phases du développement de leurs institutions sociales... La population abyssine proprement dite, issue de la fusion d'éléments chamitiques et sémitiques, reflète dans ses traditions et ses usages sociaux les habitudes particulières aux deux races, notablement modifiées d'abord par le mélange et ensuite par l'introduction du christianisme". ${ }^{28}$

El impacto de la concepción de Rossini respecto a la población de Eritrea es evidente en que, dos décadas después de publicada su obra, es retomada por la Enciclopedia italiana di scienze, lettere ed arti de 1932:

"Il Conti Rossini divide le popolazioni non tigrine della Colonia Eritrea, dal punto di vista giuridico-sociale, in due gruppi: l'uno con "ordinamento aristocratico" (gnuppo che include quasi tutte le genti eritree) l'altro con "ordinamento democratico"... L'origine etnica delle stirpi nobili sembra, anche qui, diversa, almeno in parte, dall'origine delle stirpi dei vassalli... Genti preesistenti nella regione e insieme alcuni degli stessi gruppi Agau immigrati furono storicamente ridotti in condizione di vassallaggio dai "nobili" delle stirpi bilene...". ${ }^{29}$

Sin embargo, una vez se ocupó mayor territorio, diferentes investigadores comenzaron a realizar u organizar estudios en el campo, con el fin de conocer la potencialidad económica de estos lugares. Como resultado se integró a la Accademia d'Italia, creada por Mussolini en 1926 e inaugurada en 1929, un instituto para el considerato sia come un'antica forma sociale non evoluta e sia come una involuzione o piuttosto degenerazione da società già meglio evolute. En: Conti Rossini, Principi di diritto consuetudinario dell'Eritrea (Roma: Tip. dell' Unione editrice, 1916).

${ }^{28}$ Jean-Baptiste Chabot, "C. Conti Rossini. Principi di diritto consuetudinario dell'Eritrea", Journal des savants, (1917): 561-563.

${ }_{29}$ Ver: Attilio Mori, Enrico Cerulli, Giuseppe Stefanini, Fabrizio Cortesi, Mario Salfi, y Ana Maria Ratti, "Eritrea, Colonia", Enciclopedia italiana di scienze, lettere ed arti, (Roma: Ist. della Enciclopedia Italiana, 1932). 
estudio del África Oriental Italiana (A.O.I). Otras instituciones también buscaron participar, pero solo muy pocas colaboraciones llegaron a concretarse. Una de estas, la más significativa, se desarrolló gracias al apoyo financiero de, entre otras instituciones, el Banco de Roma y el Banco de Italia, siendo dirigida por el geógrafo y paleontólogo Giotto Dainelli. La expedición, que duró siete meses, se desarrolló en la región del lago Tana en noviembre de 1936, debido al rumor de que a pesar de ser un territorio remoto, presentaba características ideales para servir al asentamiento de granjeros blancos. Entre los participantes de la expedición se encontraba Lido Cipriani, gran conocedor de la antropología y la etnología africana, quien ya había participado en varias expediciones al continente. Los resultados de la expedición empezaron a publicarse en 1938, pero debido a la cercanía de la guerra y a los resultados de esta para Italia, su impacto se vio drásticamente minimizado. Después de esta expedición, los aspectos antropológicos y etnográficos de las colonias siguieron siendo estudiados, aunque en proporción mucho menos significativa. Sin embargo, gracias a estas investigaciones se empezó a dar razón del intrincado entramado social que presentaba la población etíope, y en las últimas décadas se ha contado con información valiosa para el estudio de los procesos de cambio social y efectos del colonialismo sobre las sociedades en esta región de África Oriental. ${ }^{30}$

\section{Giuseppe Sergi}

Una figura especialmente significativa para la antropología del periodo del colonialismo fue Giuseppe Sergi, quien dedico varios años a la tarea de investigación y clasificación de las sociedades humanas. Su abundante trabajo en este ámbito le permitió desarrollar una teoría que con el tiempo fue considerada amenazadora para el régimen fascista, inclinado a la interpretación alemana, por proponer la idea de superioridad italiana frente a la africana.

Tras interesarse inicialmente por la psicología, Giuseppe Sergi (1841-1936), pasó a ocupar la catedra de Antropología en la Universidad de Bolonia en 1880. En 1884

\footnotetext{
${ }^{30}$ Roberto, “Gli scienziati italiani e la guerra d'Etiopia”, 36.
} 
TEMPUS. Revista en Historia General Medellín (Colombia), 2018, septiembre-octubre, número 8

ISSN: 2422-2976

pasó a la Universidad de Roma y en dicha ciudad fundó el Instituto de Roma y el Museo de Antropología. Fue un escritor prolífico cuya obra fue traducida al francés, el alemán, el inglés y el español. Tal como afirma Giovanni Cerro, Sergi fue uno de los más importantes antropólogos del periodo positivista, centrando sus intereses en temas como la degeneración, la eugenesia y la raza. ${ }^{31}$

En el campo de la antropología física, Sergi estableció un método morfológico para la clasificación de los grupos étnicos. Gracias a este trabajo, sentó las bases de su teoría sobre la raza mediterránea, que tendría raíces africanas, la cual enfrentó a las tesis "nordicista", según la cual los pueblos europeos eran de origen ario, y los pueblos del mediterráneo habrían perdido su pureza al haberse mezclado. Estas ideas fueron desarrolladas principalmente en su libro "La Razza Mediterranea" publicado en 1895.

En el texto, Sergi llama a la raza mediterránea: "la raza más grandiosa en el mundo" y argumenta que esta raza habría sido la responsable de las grandes civilizaciones de la antigüedad, incluyendo las del Antiguo Egipto, la Antigua Grecia y la Antigua Roma. Sergi rechazaba las tipologías raciales existentes en su tiempo, que identificaban a los pueblos mediterráneos como "blancos oscuros", porque esta concepción implicaba una visión nordicista, según la cual los pueblos mediterráneos descendían de gente de raza nórdica que se había mezclado con gente de otras razas. Sergi consideraba falsa la teoría nordicista y la rechazaba por considerarla "supremacista", pero irónicamente no tenía problemas en considerar a la "raza mediterránea" en una posición especial, reivindicando un tipo de supremacismo mediterráneo. Para el científico italiano, la raza mediterránea era una raza autónoma, de la cual habría descendido la raza nórdica. De acuerdo a esta hipótesis, la raza nórdica sería producto de una despigmentación que las poblaciones mediterráneas habrán experimentado al trasladarse a Europa del Norte. ${ }^{31}$

\footnotetext{
${ }^{31}$ Giovanni Cerro, "Giuseppe Sergi. The portrait of a positivist scientist", Journal of Anthropological Sciences (Istituto Italiano di Antropologia) 95 (2017): 1-28.

${ }^{31}$ Giuseppe Sergi, The Mediterranean Race: A Study of the Origins of the european Peoples (London: Walter Scott, 1901).
} 
Las tesis que proponía eran de por si desafiantes para su tiempo, y lo siguieron siendo durante varias décadas, siendo objeto de acalorados debates entre 1938 y 1942. En el Manifesto degli scienziati razzisti, de 1938, Mussolini y Guido Landra atacaban la teoría de Sergi, definiendo "raza" en términos puramente biológicos, sin relación a fenómenos históricos, lingüísticos o religiosos, y pretendían demostrar un origen “ario" para la población italiana, con lo que se decantaban por la interpretación del nazismo. El régimen fascista consideraba igualmente peligrosas para el imperialismo italiano las teorías cerca de un origen semita y hamitico para la "raza mediterránea". Estas ideas iban en contra de la misma tradición antropológica italiana, siendo que el mismo Guido Landra había trabajado con Sergi y defendido su mediterranismo, pasando a defender las teorías del antropólogo alemán Eugen Fisher solo cuando se vinculó al régimen.

Así, tras la publicación del Manifesto, la antropología italiana se dividió en dos ramas. Una que apoyaba el "racismo biológico", reunidos en torno a La Difesa della razza e incluía a antropólogos como Lidio Cipriani y Giovanni Marro; y otra (de los cuales el más destacado era Julius Evola) que consideraba a Sergi como el padre de la antropología italiana, si bien criticaban aspectos de su trabajo por considerar a los italianos como no-arios, y por incluir a los judíos, etíopes y bereberes dentro de los pueblos mediterráneos. Con todo, tal como afirma Giovanni Cerro, aun hubo quienes apoyaron las ideas de un racismo "nacional" o "mediterráneo" (tales como Giuseppe Genna, profesor de la Universidad de Florencia), reunidos en torno a la publicación Razza e Civiltà, que sin embargo criticaban la inclusión de pueblos contemporáneos de África Oriental y del norte en un mismo grupo con los italianos. ${ }^{32}$

Como se observa, las ideas de Sergi ejercieron una fuerte influencia, ya fuera para ser criticadas o usadas con fines más bien utilitaristas, según la política del régimen. Esto hizo que estas fueran malinterpretadas, proyectándose sobre el antropólogo la sombra del fascismo. Con todo, sus ideas marcaron un momento importante en la historia de la antropología italiana y del continente europeo, en un momento en el cual la importancia del trabajo de campo aún no había alcanzado el papel metodológico cen-

${ }^{32}$ Cerro, "Giuseppe Sergi. The portrait of a positivist scientist", 1-28. 
TEMPUS. Revista en Historia General Medellín (Colombia), 2018, septiembre-octubre, número 8

ISSN: 2422-2976

tral que posteriormente alcanzaría, ${ }^{33}$ y en donde las grandes ideas aún tenían aspiraciones universalistas, aspecto muy conveniente para los proyectos de expansión imperial.

\section{Consideraciones finales}

Como se observa en el texto, la experiencia colonial marcó el periodo inicial de desarrollo de la antropología italiana. En un primer momento los antropólogos manifestaron más bien poco interés por las realidades coloniales, al punto que los estudios necesitados por el Estado se basaron en informaciones parciales y estereotipadas, producto del trabajo de funcionarios y no de académicos. Sin embargo, posteriormente la política del régimen fascista colocó los estudios antropológicos en el centro del debate público y académico, debido a la necesidad de justificar ideológicamente las acciones del Estado y la expansión imperial.

En dicho sentido, aunque la relevancia política de la antropología fue reconocida, dicha centralidad no repercutió en una profundización o mejor obtención de los datos de campo. Esto se refleja en que las expediciones científicas a África Oriental fueron más bien escasas y de alcances limitados. Ello a pesar de que se pensaba que la antropología era una ciencia colonial por excelencia, en la medida en que brindaba a los pueblos avanzados los mecanismos para imponer su visión sobre otros pueblos en un estado inferior de desarrollo, posibilitando su ingreso tutelado a la civilización y a participar de los beneficios del "progreso".

Aunque acaso no sea un caso único, el impacto inicial de la antropología en el caso italiano fue más interno, participando en la construcción de una identidad nacional durante los periodos liberal y fascista, y siendo más bien reducido su impacto al interior de las colonias, en donde se adoptaban medidas que facilitaran el control, pero sin buscar grandes cambios en las estructuras sociales.

${ }^{33}$ En un momento más bien tardío, autores como Enrico Morselli y Lamberto Loria llamaron la atención acerca de la utilidad de los datos etnográficos para organizar de manera más efectiva las colonias y para evitar desastres como el de Adowa, pero no lograron mayor influencia. Ver: Patrizia Palumbo, A Place in the Sun. Africa in Italian Colonial Culture from Post-Unification to the Present, (Berkeley \& Los Angeles: University of California Press, 2003). 
Además de la precariedad de que hizo gala usualmente el Estado italiano en la organización efectiva de las colonias, la centralidad política que se dio a la política colonial basada en un discurso antropológico, hizo que tras la caída del régimen fascista dicha relación fuera ignorada, e incluso ocultada, siendo apenas recatada y reevaluada en las últimas décadas. Con todo, la experiencia colonial pasó por ser el contexto en el cual surgió y se desarrolló una escuela antropológica italiana, de allí que, pese a los avances realizados, sus verdaderas implicaciones sobre dicho campo de estudio aún están por ser establecidas.

\section{Bibliografía}

Ben-Ghiat, Ruth, y Mia Fuller. Italian Colonialism. Nueva York: Palgrave Macmillan, 2005.

Benini, Rodolfo. "Le ragioni dell'Italia". Giornale degli economisti, (1936): 1-4.

Burbank, Jane, y Frederick Cooper. Empires in world history. Power and the politics of difference. Princeton: Princeton University Press, 2010.

Cerro, Giovanni. "Giuseppe Sergi. The portrait of a positivist scientist". Journal of Anthropological Sciences (Istituto Italiano di Antropologia), 95 (2017): 1-28.

Chabot, Jean-Baptiste. "C. Conti Rossini. Principi di diritto consuetudinario dell'Eritrea". Journal des savants, (1917): 561-563.

Duggan, Christopher. Historia de Italia. Cambridge: Cambridge University Press, 1996.

Goberna Flaque, Juan. "What's culture: Cien años de controversias en la antropologia anglosajona (1871-1971)”. Gallaecia, ${ }^{\circ} 22$ (2003): 531-554.

Harris, Marvin. El desarrollo de la teoria antropologica: Una historia de las teorias de la cultura. Madrid: Siglo Veintiuno de España Editores, 2005.

Lanternari, Vittorio. Antropologia e Imperialismo. Turin: Einaudi, 1974.

Morgan, Lewis. La Sociedad Primitiva. Buenos Aires: Lautaro, 1946. 
TEMPUS. Revista en Historia General Medellín (Colombia), 2018, septiembre-octubre, número 8 ISSN: 2422-2976

Mori, Attilio, Enrico Cerulli, Giuseppe Stefanini, Fabrizio Cortesi, Mario Salfi, y Ana Maria Ratti. "Eritrea, Colonia”. En Enciclopedia italiana di scienze, lettere ed arti, de Ist. della Enciclopedia Italiana. Roma: Ist. della Enciclopedia Italiana, 1932.

Palumbo, Patrizia. A Place in the Sun. Africa in Italian Colonial Culture from PostUnification to the Present. Berkeley \& Los Angeles: University of California Press, 2003.

Panza, Valerio. La colonizzazione giuridica dell'Eritrea: diritto coloniale tra scienza giuridica, antropologia, etnografia giuridica (1880-1912). Nápoles: Università Degli Studi Di Napoli "Federico II", 2014.

Pavone, Caludio. "The Two Levels of Public Use of the Past". Mediterranean Historical Review, no 16 (2001): 74-86.

Ranjan, Priyansh. "Edward Said's 'Orientalism': A Post-Colonial Culture Study". IOSR Journal Of Humanities And Social Science 20, no 9 (2015): 85-88.

“Razzismo Italiano”. La Difesa della Razza 1, n 1 (1938): 1.

Roberto, Maiocchi. “Gli scienziati italiani e la guerra d'Etiopia”. Culture Economie e Territori, $\mathrm{n}^{\mathrm{o}} 32$ (2012).

Rossini, Conti. Principi di diritto consuetudinario dell'Eritrea. Roma: Tip. dell' Unione editrice, 1916.

Sergi, Giuseppe. The Mediterranean Race: A Study of the Origins of the european Peoples. London: Walter Scott, 1901.

Sorgoni, Barbara. "An Italian officer in colonial Eritrea: administration, ethnography and gender". En Ordering Africa. Anthropology, European imperialism and the politics of knowledge, de H. Tilley y R. J. Gordon, 330-374. Manchester: Manchester University Press, 2007. 
Diego Giovanni Castellanos

. "Italian anthropology and the Africans: the early colonial period". En $A$ Place in the Sun. Africa in Italian Colonial Culture from Post-Unification, de Palumbo, 62-80. Berkeley / Los Angeles: University of California Press, 2003.

Stefanini, Giuseppe. “Assab”. En Enciclopedia italiana di scienze, lettere ed arti, de Istituto Giovanni Treccani. Roma: Istituto della Enciclopedia italiana, 1929.

Tylor, Edward. Cultura Primitiva: Los orogenes de la cultura. Madrid: Ayuso, 1977.

Volpe, Charlotte. Imagined Proximities: The Use of History and Geography as Rhetorical Justifications for Italian Colonial Conquest in Tripolitania. Nueva York: Barnard College, 2014. 